\title{
Alfabetización Estadística del profesorado. Una revisión sistemática de 2010 a 2020
}

| Faculty Statistical Literacy of teachers. A systematic review from 2010 to 2020 |

\author{
Adriana Jaqueline Avilez Poot \\ A07001469@alumnos.uady.mx \\ Universidad Autónoma de Yucatán \\ Yucatán - México
}

\author{
(D) Alfredo Zapata González \\ zgonza@correo.uady.mx \\ Universidad Autónoma de Yucatán \\ Yucatán - México
}

Resumen: Este análisis consiste en una revisión sistemática para conocer el estado de las investigaciones relacionadas con la alfabetización estadística enfocado en el profesorado en los últimos diez años. Para el estudio se revisaron 606 artículos de nueve bases de datos académicas, asimismo, se utilizaron criterios de inclusión y exclusión y se obtuvieron 16 fuentes para el análisis. Entre los resultados, se destaca que ha habido un incremento en las investigaciones enfocadas hacia una evaluación del conocimiento estadístico de los profesores y un desarrollo de la alfabetización estadística. Es importante seguir fomentando investigaciones bajo esta temática pues es deseable que todo docente cuente con una alfabetización estadística para desenvolverse en un mundo globalizado en datos.

Palabras Clave: Alfabetización Estadística, Profesores, Educación Estadística, Revisión sistemática

Abstract: This analysis consists of a systematic review to know the state of research related to statistical literacy focused on teachers in the last ten years. For the study, 606 articles from nine academic databases were reviewed, likewise, inclusion and exclusion criteria were used and 16 sources were obtained for analysis. Among the results, it stands out that there has been an increase in research focused on an evaluation of the statistical knowledge of teachers and a development of statistical literacy. It is important to continue promoting research under this theme as it is desirable that all teachers have statistical literacy to function in a world globalized in data.

Keywords: Statistical Literacy, Teachers, Statistical Education, Systematic review

\section{Introducción}

La estadística se ha caracterizado por ser una herramienta multidisciplinaria ya que su utilidad se refleja en todas las áreas, además es fundamental para la toma de decisiones que influyen en el desarrollo de los países, desde lo histórico, cultural, económico, político y social, pues de acuerdo con diversos autores como Batanero (2002), Tauber, Cravero y Redondo (2013), la información generada mediante el uso de la estadística ayuda a tomar decisiones en un sentido crítico que sustenten el éxito de algún fenómeno o situación. De acuerdo con lo anterior, se puede afirmar que los ciudadanos 
reciben y se encuentran dentro de una sociedad que hace uso de información que requiere de la estadística en gran medida y en consecuencia se presenta la necesidad e importancia de apostar por su enseñar, siendo necesario una educación estadística desde los primeros niveles educativos hasta niveles de posgrado donde la vinculación con la investigación es fundamental, de esta manera se podría tener un conocimiento suficiente para leer e interpretar resúmenes estadísticos.

Sin embargo, desde inicios del siglo XXI, investigaciones como las de Batanero (2001), Batanero (2002), Estrada (2002), Tauber (2012), señalan que tanto de manera política, curricular y en la práctica de los docentes, la educación estadística ha sido relegada, ya que si bien aparece el contenido estadístico en el currículo de educación básica muchas veces son los docentes quienes evitan su enseñanza, por lo que es necesario realizar estudios que impulsen la importancia de esta área. Por su parte, BenZví y Garfield (2004), afirman que la información cuantitativa está en todas partes y la estadística continuamente es presentada como una forma de agregar credibilidad a los anuncios, argumentos o propagandas, requiriendo con ello de una alfabetización estadística para ser capaz de evaluar apropiadamente la evidencia y realizar conclusiones basadas en los datos; de esta manera y de acuerdo a lo señalado por Batanero (2002), entendemos por alfabetización estadística tanto el conocimiento básico como las habilidades que toda persona debe tener para leer e interpretar datos estadísticos, de tal manera que pueda comprender la información que se presenta en una sociedad y que influyen en sus vidas.

Dicha alfabetización estadística debe fomentarse como una de las prioridades en los docentes de cualquier área y nivel educativo, debido a que resulta importante su uso para tomar decisiones. Por ejemplo, a partir de los resultados de las calificaciones de los estudiantes, las evaluaciones otorgadas a su desempeño como docente e incluso tomar decisiones administrativas.

Es por ello por lo que la presente revisión sistemática de la literatura tiene como objetivo analizar las investigaciones realizadas acerca de la alfabetización estadística en el profesorado tanto de nivel básico como universitario entre los años 2010 a 2020, considerando la siguiente pregunta central de la investigación: ¿Cuáles son los factores asociados a la alfabetización estadística que se han evaluado con mayor frecuencia en el profesorado?

Cabe destacar que la relevancia de esta investigación se hace evidente dado que solo se encontró un estudio de revisión sistemática relacionada con la alfabetización estadística, el cual se centra en el desarrollo de las habilidades de alfabetización estadística de los estudiantes, y fue publicado por Aziz y Rosli (2021). En este estudio se analizaron 36 artículos que se enfocan en la temática de interés, obteniendo como resultado principal la identificación de los métodos de enseñanza que influyen en el desarrollo de la competencia estadística entre los estudiantes. De acuerdo con la revisión de la literatura realizada, no se encontró ninguna revisión sistemática centrada en los docentes.

\section{Revisión sistemática}

De acuerdo con Jesson, Matheson y Lacey (2011) la revisión sistemática es definida a manera de un método de investigación que culmina en un producto escrito ya sea como parte de un proyecto de investigación, de una tesis o de manera independiente, no obstante, su finalidad es producir hallazgos.

Por otra parte, Richter, Kerres, Bedenlier y Bond (2020) la definen como un artículo académico en el cual se proporciona un panorama general del conocimiento actual sobre un tema y por tanto también produce conocimiento, en consecuencia, a determinar y esclarecer lo que hay y lo que no hay de un fenómeno, pues también se determinan aquellos vacíos por investigar. Asimismo, dichos autores concuerdan en que las revisiones sistemáticas se basan en una metodología rigurosa para obtener la información actual del tema y más pura del fenómeno estudiado de acuerdo con criterios que delimiten el tema de interés. 


\section{Marco metodológico}

\subsection{Concepción metodológica de la investigación}

Para efectos del sentido de esta investigación, se seleccionó la metodología de revisión sistemática definida por Jesson et al. (2011), quienes consideran que está es aquella que produce hallazgos de un tema en particular, en el que se utiliza un enfoque metodológico sistemático prescrito, al revisar la literatura y las investigaciones realizadas respecto a la misma, a través de los siguientes pasos: 1) Definir la pregunta de investigación, 2) Diseñar un plan, 3) Localización de la literatura, 4) Aplicar criterios de exclusión e inclusión, 5) Aplicar evaluación de calidad y 6) Escribir la síntesis. Se eligió esta metodología al ser un primer acercamiento para conocer el panorama actual de la temática de interés y que al mismo tiempo al ser rigorosa, pueda ser reproducible, asimismo considerando el resultado de Aziz y Rosli (2021) en el que se señala las pocas investigaciones relacionadas con la temática de alfabetización estadística de manera general, por lo que no se encontró pertinente seguir la metodología de un metaanálisis.

Los motores de búsqueda utilizados en la revisión de las investigaciones fueron Dialnet, EBSCO, Redalyc, JSTOR, SAGE, SCOPUS, ScienceDirect y Google Scholar. En cuanto a las cadenas de búsqueda se usaron las siguientes palabras clave tanto en español como en inglés: alfabetización estadística, profesores, educación estadística; asimismo se aplicaron los conectores boléanos AND y OR para unir sinónimos, por lo que se generó las siguientes cadenas de búsqueda:

- "alfabetización estadística" AND (profesores OR docentes OR profesorado) AND "Educación Estadística"

- "statistical literacy" AND (Univeristy OR college OR higher OR primary school) AND (faculty OR teachers OR professor OR instructor)

En la tabla 1 se presenta la relación de los criterios de inclusión y exclusión que se tomaron en cuenta.

Posteriormente, una vez definidos los criterios se inició con el paso de aplicar la evaluación de calidad de cada artículo, dicho proceso se describe en la figura 1 según la metodología de PRISMA (2020).

En dicha figura se evidencia que, de las 9 bases de datos académicas distintas, se obtuvo 606 fuentes tanto en inglés como en español, de los cuales después de analizar y quitar las fuentes duplicadas y una vez aplicados los criterios de inclusión y exclusión se mantuvieron únicamente 14 artículos y 2 capítulos de libro.

\section{Resultados}

En la figura 2 se presentan los resultados del análisis realizado a las 16 fuentes después de aplicar los criterios de inclusión y exclusión.

A partir de esta figura, se evidencia que en el último año ha habido un creciente interés en investigar la alfabetización estadística de los profesores en ejercicio, ya que de las 16 investigaciones analizadas siete corresponden al año 2020, seguido de tres del año 2018, en los demás años han habido al menos una publicación por año excepto en el 2012, 2013 y 2019 donde no se encontraron investigaciones relacionadas con el tema, esto rectifica lo reportado en la revisión sistemática elaborada por Aziz y Rosli (2021) quienes a pesar de enfocarse en la alfabetización estadística de los estudiantes también reportan el poco interés en investigar bajo esta línea de investigación.

De manera global, se reporta que de las 16 fuentes analizadas se encontró que la mayoría son acciones investigativas con metodología cuantitativa con nueve investigaciones, siendo generalmente estudios 
Tabla 1: Criterios de inclusión y exclusión. Fuente: Elaboración propia.

\begin{tabular}{l} 
INCLUSIÓN \\
\hline Año de publicación 2010-2020. \\
Artículos empíricos, capítulos de libro \\
Profesores cualquier nivel educativo básico \\
(preescolar, primaria, secundaria), medio su- \\
perior y superior.
\end{tabular}

El artículo contiene las palabras clave establecidas.

Idiomas: Español, inglés.

Se explicita desde el resumen qué, cómo, dónde, cuándo y con quiénes se realizó el estudio.

Se señala la metodología de investigación de manera clara, es decir, se puntualiza si la investigación es cuantitativa o cualitativa.

Se puntualizan los resultados obtenidos.

La investigación clarifica y explica la conceptualización de alfabetización estadística de la cual se basa.
EXCLUSIÓN

Artículos diferentes del rango de años establecido.

Memorias, libros y actas de congresos, tesis, artículos no empíricos.

Estudiantes, otros profesionistas.

El artículo no contiene las palabras clave establecidas.

Artículos que no estén en idioma español o inglés.

No se explicita o no es claro desde el resumen qué, cómo, dónde, cuándo y con quiénes se realizó el estudio.

No señala de manera clara la metodología de investigación.

El artículo no presenta los resultados.

No se explica o no es clara la conceptualización de alfabetización estadística empleada.

en los que se aplica un instrumento para analizar la alfabetización estadística de los profesores o incluso considerando también evaluar las percepciones y las actitudes, así como la relación entre dichas variables. En contraste, en una menor medida se encontraron cuatro investigaciones de metodología cualitativa, dichos estudios se centraron en analizar observaciones de clases, entrevistas y revisión del material de enseñanza y aprendizaje de la estadística, así como estudios de caso. Adicionalmente, se destaca que se encontraron tres estudios considerados mixtos, debido a que analizan la alfabetización estadística con instrumentos que miden el conocimiento pero también se hace uso de entrevistas y observaciones de campo para triangularizar la información, si bien los autores señalan que la metodología es mixta, solo hacen uso de instrumentos y estrategias propias de la metodología cualitativa como la observación, las entrevistas individuales y grupos de enfoque. Sin embargo, los resultados sirven para describir a la población de estudio y determinar inferencias en torno a ello.

Por otra parte, se destaca que las investigaciones se pueden clasificar de acuerdo con los objetivos de estudio de la siguiente manera:

- Cinco de los estudios se centran en evaluar la alfabetización estadística o contenidos de ella, como gráficos, medidas de tendencia central y problemas de probabilidad y variabilidad, en todos los estudios se concluyen que los docentes requieren de una capacitación para desarrollar y potenciar el conocimiento estadístico; estas investigaciones corresponden a las elaboradas por (Cuevas y Ramírez, 2018; Engledowl y Tarr 2020; Mantilla y Estrada, 2020; Rodríguez, Díaz y Vásquez, 2018; Yumi, Castro, Souza, Rodrigues y Oliveira, 2011). 
Identificación de estudios vía base de datos y registros

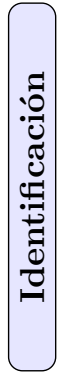

\begin{tabular}{|l|l|}
\hline $\begin{array}{l}\text { Registros identificados en } \\
\text { búsqueda de la base*: } \\
\text { base de datos }(n=9) \\
\text { Registros }(n=606)\end{array}$ & $\begin{array}{l}\text { Registros eliminados antes } \\
\text { de la selección: } \\
\text { Registros duplicados } \\
\text { eliminados }(n=4) \\
\text { Registros removidos por } \\
\text { otras razones }(n=520)\end{array}$ \\
\cline { 2 - 2 }
\end{tabular}

\begin{tabular}{|l|l|}
$\begin{array}{l}\text { Registros seleccionados } \\
(n=82)\end{array}$ & $\begin{array}{l}\text { Registros excluidos** } \\
(n=524)\end{array}$ \\
\hline
\end{tabular}

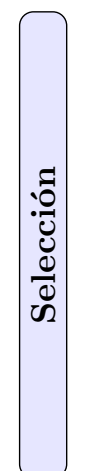
Registros seleccionados a base de título y resumen $(n=27)$

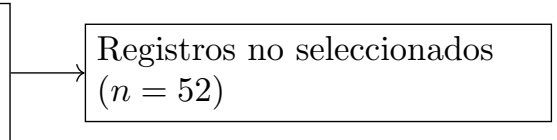

Registros seleccionados a base de revisión de texto completo (criterio elegibilidad) $(n=16)$

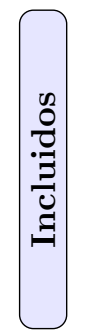

$\longrightarrow \begin{aligned} & \text { Registros no seleccionados } \\ & (n=11)\end{aligned}$

Figura 1: Secuencia del proceso de revisión. Fuente: PRISMA (2020).

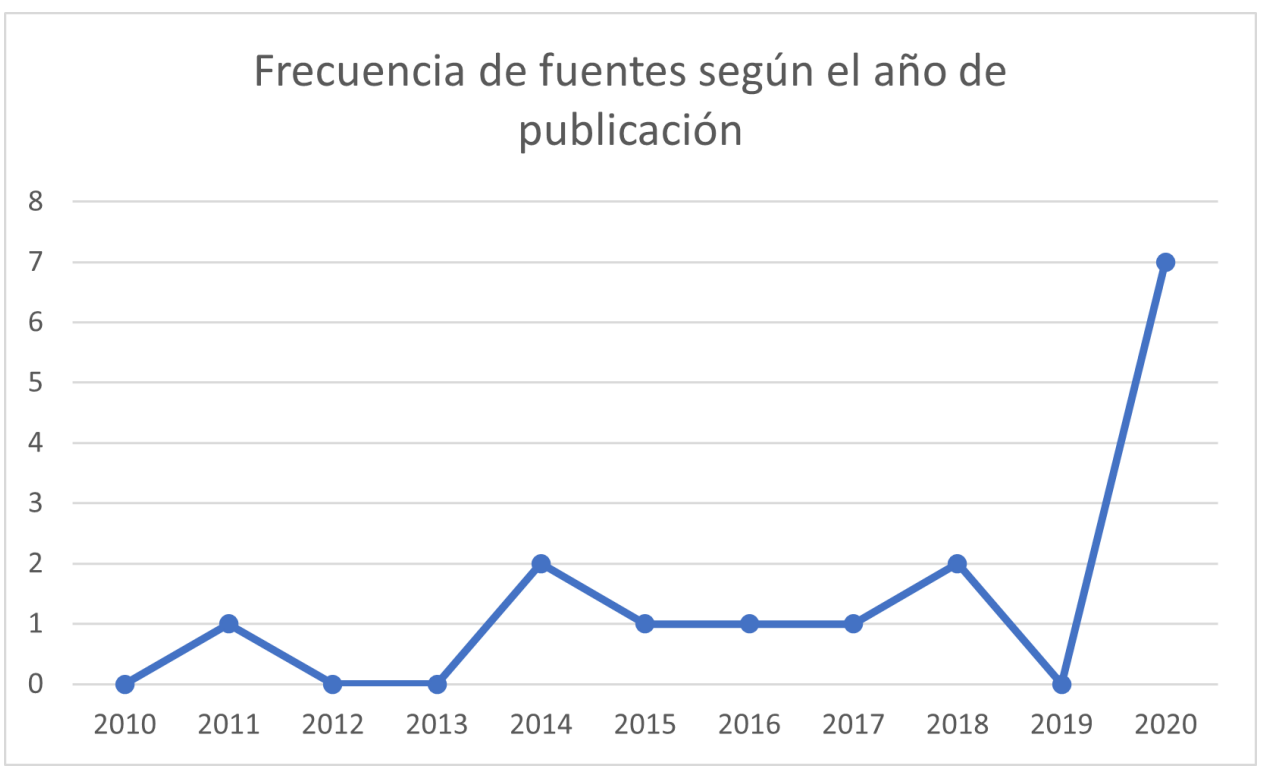

Figura 2: Frecuencia de artículos según el año de publicación. Fuente: Elaboración propia. 
- Cuatro de los estudios analizados se han enfocado en desarrollar una alfabetización estadística en los docentes, es decir son investigaciones que buscan que el docente potencie ciertas habilidades o desarrolle algún componente de la alfabetización estadística por medio de actividades, dinámicas o técnicas. Dichos estudios corresponden a las investigaciones de (García, Fernández e Imilpán, 2020; Muñiz y Rodríguez, 2020b; Muñiz, Rodríguez y Alsina, 2020c; Zapata-Cardona, 2020); incluso los estudios de Muñiz y Rodríguez corresponden a un mismo proyecto que fueron desarrollando paulatinamente.

- Dos estudios evalúan tanto conocimiento como actitudes hacia la estadística, por lo que también se analiza una posible relación entre estos. Dichos estudios, elaborados por Loewen, Lavolette, Spino, Papi, Schmidtke, Scott y Wolff (2014) y Rodríguez (2017), consideran que las actitudes juegan un papel importante para la adquisición y buen uso del conocimiento estadístico.

- Dos estudios se enfocan en evaluar las perspectivas sobre el aprendizaje y la enseñanza de la estadística en términos de alfabetización estadística. Estas investigaciones desarrolladas por $(\mathrm{Mu}-$ ñiz y Rodríguez, 2020a; Oliveira y Caetano, 2018), determinan que los profesores siguen la tendencia de priorizar asignar actividades de libros de texto, cuyos ejercicios no están contextualizados por lo que es difícil desarrollar componentes como el sentido estadístico.

- También se encontró un estudio en cada uno de los siguientes temas: análisis de evaluaciones realizadas por los docentes (Salcedo, 2014), cambios de creencias hacia la estadística (Olfos, Estrella y Morales, 2015) y evaluación sobre el conocimiento estadístico y didáctica (Hazelton, Allen, MacLeod, LeBlanc y Boudreau, 2016).

Cabe destacar, que todos los estudios reiteran la necesidad de continuar con la investigación en el tema, puesto que se evidencia un área de oportunidad para subsanar las brechas existentes en cuanto a la alfabetización estadística de los docentes.

En la figura 3, se presentan los resultados relacionados al nivel educativo de los docentes que han participado en los estudios analizados. Se obtuvo que las investigaciones se han enfocado en su mayoría a profesores de nivel superior (6) siendo los estudios de Hazelton et al. (2016), Loewen et al. (2014), Oliveira y Caetano (2018), Rodríguez (2017), Rodríguez-Alveal, Díaz-Levicoy, Vásquez-Ortiz (2018) y Salcedo (2014). Además, la mayoría de las investigaciones han sido realizadas considerando a profesores que enseñan Matemáticas excepto las investigaciones de Loewen et al. (2014) que se enfoca en docentes que enseñan lingüística y el estudio de Hazelton et al. (2016) centrado en profesores de Medicina que enseñan estadística.

Posteriormente, los estudios de Muñiz y Rodríguez (2020a), Muñiz y Rodríguez (2020b) y Muñiz y Rodríguez (2020c) se enfocan en analizar la alfabetización estadística de los profesores de nivel Secundaria que enseñan Matemáticas. En cuanto a investigaciones centradas en el profesorado tanto de Secundaria como de Bachillerato, se destacan las propuestas de Cuevas y Ramírez (2018), Engledowl y Tarr (2020). Con respecto a las investigaciones centradas en profesores de nivel Primaria, se cuenta con los estudios de Olfos, Estrella, Morales (2015) y Yumi et al. (2011).

También, se comenta que el único estudio que abarca profesores de los niveles educativos Secundaria y Primaria corresponde a Zapata (2020). La investigación de García, Fernández y Imilpán (2020), es el único estudio centrado en profesores de los tres niveles de educación básica (Primaria, Secundaria y Bachillerato). Por último, se cuenta con un estudio centrado en profesores de bachillerato de los autores Mantilla y Estrada (2020).

Por otra parte, se obtuvo como resultado que 12 de los 16 estudios analizados se desarrollaron en países de América, destacando Brasil con 3 contribuciones, Chile, México, EUA con 2 publicaciones cada uno y por último Canadá, Colombia y Ecuador con un artículo por país. Solo tres de las investigaciones se realizaron fuera del continente Americano, de manera específica en España, siendo en los últimos años los principales pioneros Muñiz y Rodríguez (2020a) Muñiz y Rodríguez (2020b) y Muñiz y Rodríguez (2020c). Lo anterior coincide con lo señalado por Gea, Álvarez y Garzón (2020) 


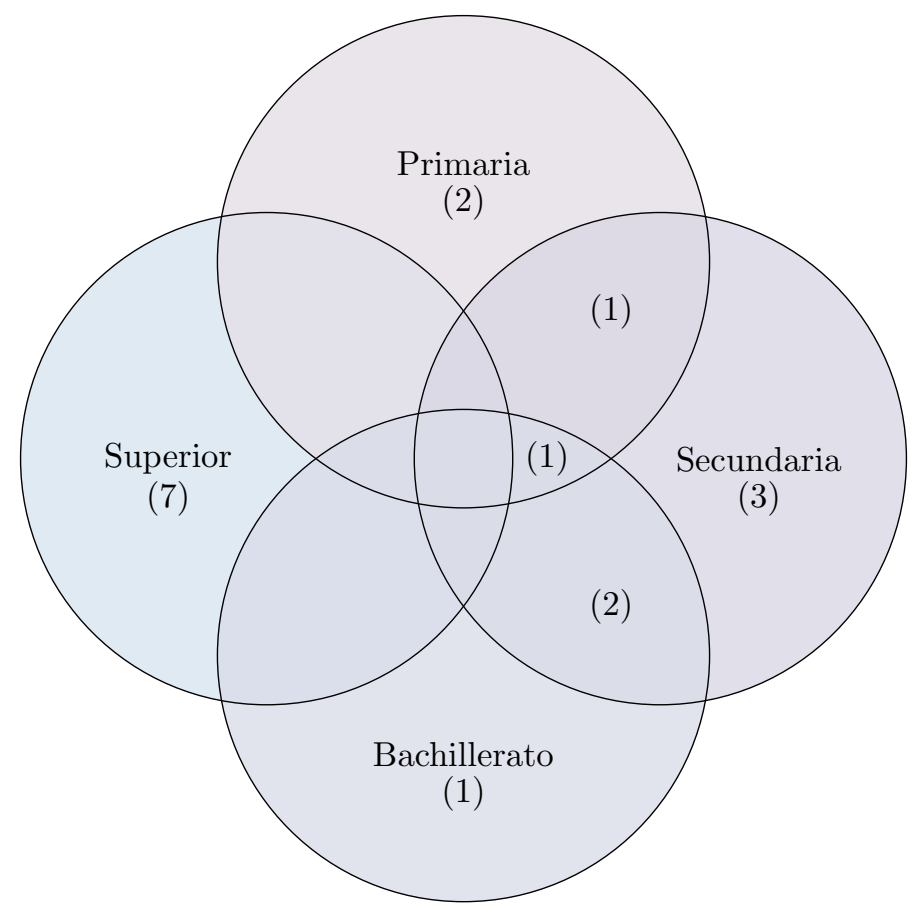

Figura 3: Nivel educativo de los docentes que han participado en los estudios analizados. Fuente: Elaboración propia.

quienes reportan que en Brasil y España existen grupos de investigación consolidados dirigidos a la educación estadística y probabilística.

En la figura 4, se observa que el mayor número de los estudios analizados se encuentran publicados en revistas con factor de impacto, los cuales en su mayoría se encuentran en el cuartil 3 (Q3), seguido de publicaciones que se encuentran en el cuartil $4(\mathrm{Q} 4)$. Un par de estudios se publicaron en revistas que se ubican en el punto más alto de la clasificación (Q1) y un estudio en el cuartil 2 (Q2). A partir de los resultados se podría inferir que, en los últimos 10 años, las revistas con factor de impacto han tenido un interés en publicar este tipo de temática. Asimismo, tres de las investigaciones se encuentran publicadas en revistas indexadas en bases de datos académicas y dos son capítulos de libros.

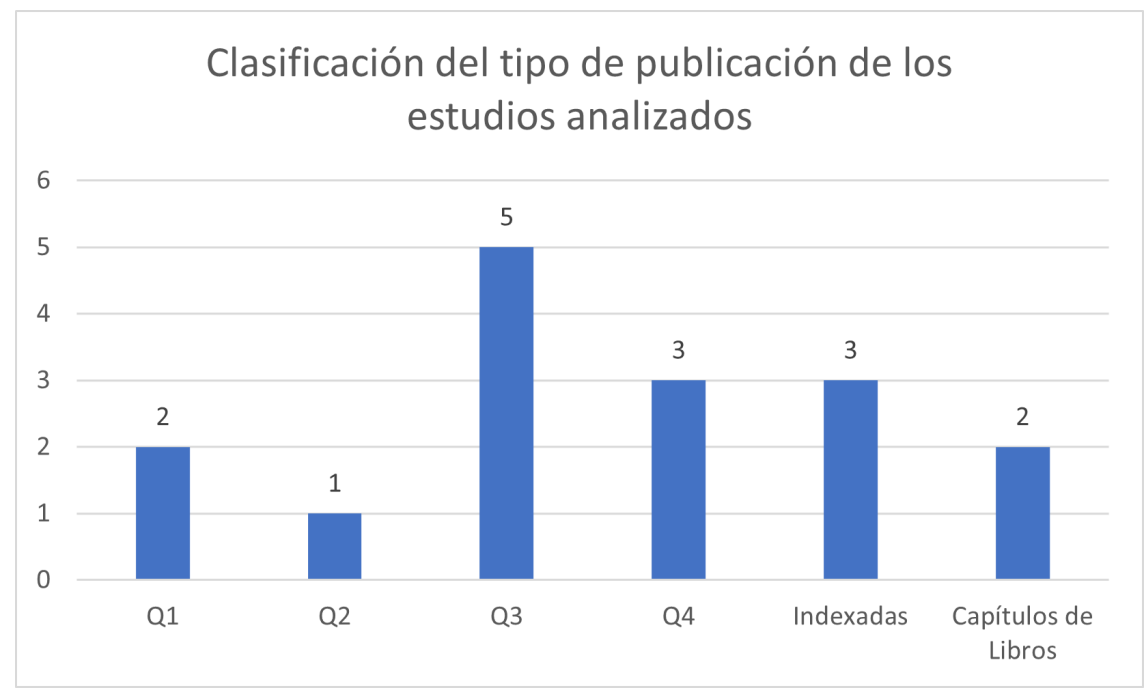

Figura 4: Clasificación del tipo de publicación de los estudios analizados. Fuente: Elaboración propia. 


\section{Conclusiones}

El análisis de las investigaciones de la revisión sistemática realizada, evidencian la necesidad inherente por realizar mayores investigaciones relacionadas a la alfabetización estadística de los docentes, ya que se considera que 14 artículos y 2 capítulos de libros de los 606 artículos iniciales en las 9 bases de datos académicas revisadas, son escasos, considerando un intervalo de tiempo de diez años, más aún, cuando diversos investigadores como Batanero (2011), Pinto, Tauber, Zapata, Albert, Ruiz y Mafokozi (2017) y Pinto y Castillejos (2020) reportan que ha existido un incremento en investigaciones en el campo de la Educación Estadística.

Aunado a lo anterior, es necesario realizar investigaciones sobre los docentes que no necesariamente tienen una formación matemática pero que hacen uso de la estadística en su día a día en el aula o como herramienta de investigación, puesto que únicamente dos de estos estudios se centraron en profesores que no son del área de matemáticas pero que reconocen la necesidad de contar con un adecuado manejo de términos estadísticos, de razonamiento estadístico y de un sentido de los datos.

En respuesta a la pregunta central de la investigación, se puede afirmar que los factores que más se han evaluado asociados a la alfabetización estadística de los profesores han sido los relacionados a la evaluación del conocimiento estadístico, al desarrollo de la alfabetización estadística y al análisis de la didáctica de la estadística, considerando en este último aspecto las percepciones hacia el aprendizaje y enseñanza de la estadística.

Por último, se reitera la importancia de realizar investigaciones enfocadas en la alfabetización estadística centradas en el docente, desde el nivel básico hasta el universitario, ya que hoy en día es indispensable contar con una alfabetización estadística para interpretar la información que se presenta todos los días en nuestro entorno.

\section{Agradecimientos}

Esta investigación ha sido posible gracias a la asignación de efectivos a través de la beca número 888116 del Consejo Nacional de Ciencia y Tecnología (CONACYT) del Gobierno de México.

\section{Bibliografía}

[1] Aziz, A. y Rosli, R. A systematic literatura review on developing students' statistical literacy skills. Journal of Physics: Conference Series. 2021.

[2] Batanero, C. Situación actual y perspectivas futuras de la didáctica de la Estadística. En Batanero, Carmen. Didáctica de la Estadística. Granada, España: Grupo de Investigación sobre Educación Estadística. 3-7. 2001.

[3] Batanero, C. Los retos de la cultura estadística. Jornadas Interamericanas de Enseñanza de la Estadística, Buenos Aires. Conferencia inaugural. 2002.

[4] Batanero, C. y Díaz, C. Estadística con proyectos. Departamento de Didáctica de la Matemática. Universidad de Granada, Granada, España. 2011.

[5] Ben-Zvi, D. y Garfield, J. Statistical Literacy, Reasoning and Thinking: goals, definitions and challenges. En: D. Ben-Zvi y J. Garfield (eds.), The challenge of developing statistical literacy, reasoning and thinking, 3-15. 2004. 
[6] Cuevas, H. y Ramírez, G. Desempeño en estocástica entre profesores de educación secundaria: un estudio exploratorio en dos regiones de Costa Rica y México. Educación matemática, 1(30), 93-132.2018.

[7] Engledowl, C. y Tarr, J. Secondary Teachers' Knowledge Structures for Measures of Center, Spread y Shape of Distribution Supporting their Statistical Reasoning. International Journal of Education in Mathematics, Science and Technology, 8(2), 146-167. 2020.

[8] Estrada, A., Batanero, C. y Fourtiny, J. Análisis de las actitudes y conocimientos estadísticos elementales en la formación del profesorado. Tesis doctoral. Universidad de Granada, España. 2002.

[9] García, J., Fernández, N. y Imilpán, I. Desarrollo del razonamiento probabilístico en profesores de matemáticas mediante simulación computacional. Revista Paradigma, (16), 404-426. 2020.

[10] Gea, M., Álvarez, R. y Garzón, J. Seminario Hispano- Brasileño de Educación Estadística. Departamento de Didáctica de la Matemática. Universidad de Granada Revista. 2020.

[11] Hazelton, L., Allen, M., MacLeod, T., LeBlanc, C. y Boudreau, M. Assessing Clinical Faculty Understanding of Statistical Terms Used to Measure Treatment Effects and Their Application to Teaching. J Contin Educ Health Prof, 4(36), 278-283. 2016.

[12] Jesson, J., Matheson, L. y Lacey, F. Doing your literature review, Tradicional and Systematic Techniques. SAGE Publications LTD. 2011.

[13] Loewen, S., Lavolette, E., Spino, L., Papi, M., Schmidtke, J., Scott, S., \& Wolff, D. Statistical Literacy Among Applied Linguists and Second Language Acquisition Researchers. TESOL Quarterly, 48(2), 360-388. 2014.

[14] Mantilla, M. y Estrada, J. Educación Estadística para profesores de bachillerato. Sinergias Educativas, 5, 19-30. 2020.

[15] Muñiz, L. y Rodríguez, L. Dificultades del profesorado de Educación Secundaria para fomentar la alfabetización estadística. Investigaçoes Hispano-Brasileiras en Educaçao Estatística, 100106. 2020a.

[16] Muñiz, L. y Rodríguez, L. Fomentando la alfabetización estadística en el profesorado de educación secundaria mediante una actividad formativa y evaluadora. Seminario Hispano Brasileño de Educación Estadística. 41-44 Granada. 2020b.

[17] Muñiz, L. y Rodríguez, L. \& Alsina, A. Deficits in the Statistical and Probabilistic Literacy of Citizens: Effects in a World in Crisis. Mathematics, 11(8). 2020c.

[18] Olfos, R., Estrella, S., y Morales, S. Clase pública de un estudio de clases de estadística Una instancia de cambio de creencias en los profesores. Revista Electrónica Educare, 3(19), 1-17. 2015.

[19] Oliveira, A. y Caetano, F. Perception of professors who teach statistics in higher education in Brazil towards statistical teaching. Acta Scientiae, 20(1), 2-19. 2018.

[20] Page, M., McKenzie, J., Bossuyt, P., Boutron, I., Hoffmann T. y Mulrow C. The PRISMA 2020 statement: an updated guideline for reporting systematic reviews. BMJ 2021;372:n71. 2020.

[21] Pinto, J. y Castillejos, A. Propuesta de una prueba de alfabetización estadística en temas de pobreza y desigualdad en México. Educación y ciencia, 9 (54), pp. 66-82. 2020.

[22] Pinto, J., Tauber, L., Zapata, L., Albert, A., Ruiz, B. y Mafokozi, J. Alfabetización Estadística en Educación Superior. Acta Latinoamérica de Matemática Educativa. Capítulo 1. Análisis del discurso Matemático Escolar. 227-235. 2017. https : //www. clame. org.mx/documentos/alme30.pdf 
[23] Richter, Kerres, Bedenlier y Bond. Systematic Reviews in Educational Research. Methodology, perspectives and application. Springer Vs. 2020.

[24] Rodriguez, F. Alfabetización estadística en profesores de Distintos Niveles Formativos. Educação \& Realidade, 42 (4), 1-19. 2017.

[25] Rodriguez-Alveal, F. Díaz-Levicoy, D. y Vásquez-Ortíz, C. Evaluación de la alfabetización probabilística del profesorado en formación y en activo. Estudios pedagógicos (Valdivia), 44(1), 135-156. 2018.

[26] Salcedo, A. Statistics Test Questions: Content and Trends. Statistics Education Research Journal, 13(2), 202-217. 2014.

[27] Tauber, L., Cravero, M. y Redondo Y. Evaluación de errores de profesores de mate matemática en tareas de Alfabetización Estadística y de Razonamiento Estadístico. I Jornadas Virtuales en Didáctica de la Estadística, Probabilidad y Combinatoria. Universidad Nacional de Litoral. Argentina. 2013.

[28] Yumi, V., Castro, A., Souza, A., Rodrigues, A. \& Oliveira, M. A educação estatística no ensino fundamental II em Lavras, Minas Gerais, Brasil: avaliação e intervenção. Revista Latinoamericana de Investigación en Matemática Educativa, 14(2), 233-263. 2011.

[29] Zapata-Cardona L. Colaboración entre Profesores de Estadística e Investigadores: Aportes al Desarrollo Profesional. Bolema, Rio Claro, 34(68), 1285-1303. 2020. 